SISOMOS AMERICANOS

Revista de Estudios

Transfronterizos
Volumen XXI, número l,

enero-junio de 2021

Recibido: 9 de noviembre de 2020

Aprobado: 21 de marzo de 2021

\title{
Paradiplomacia y biodiplomacia: formas plurales de negociar la vida
}

\section{Paradiplomacy and biodiplomacy: Pluralistic forms of negotiating life}

\author{
Dennis Sorondo Salazar* \\ Universidad del País Vasco, España.
}

\begin{abstract}
Resumen
El COVID-19 ha condicionado nuestras formas de ser y vivir. La diplomacia, como mediación del extrañamiento y como forma plural de negociar la vida, tampoco ha sido ajena a los cambios generados por la pandemia. Este artículo amplía el alcance de la diplomacia y la paradiplomacia para introducir en el debate el concepto de biodiplomacia y exponer las posibilidades de análisis que hace emerger. Es así como mediante el análisis de este concepto surgen múltiples intersecciones y espacios intermedios a partir de técnicas de gobierno, formas de negociar la vida y prácticas diplomáticas. Se recurre a la biodiplomacia para analizar las formas plurales de mediación del extrañamiento y la negociación de la vida que se manifiestan en los espacios liminales. La línea argumental de este texto apunta a que la biodiplomacia puede servir para examinar la forma en que los sujetos "negocian su vida" y su "vida es negociada" junto y en relación con otros múltiples organismos y seres.
\end{abstract}

Palabras clave: paradiplomacia, biodiplomacia, negociación de formas de ser y vivir.

\footnotetext{
* Doctor en Estudios Internacionales, Facultad de Ciencias Sociales y de la Comunicación, Universidad del País Vasco. Dirección: Euskal Herriko Unibertsitatea, Barrio Jauregi, Casa Ikusmen Alaia 39, España, Gipuzkoa, Hernani, 20120. Correo electrónico: dennisorondo18@gmail.com ORCID: https://orcid.org/0000-0002-8882-8560
} 


\begin{abstract}
The COVID-19 disease has conditioned our ways of being and living. Diplomacy, as a mediation of estrangement and as a pluralistic way of negotiating life, has not been immune to the changes generated by the pandemic. This article broadens the scope of diplomacy and paradiplomacy to introduce the concept of biodiplomacy and reveal the possibilities for analysis that it raises. The analysis of this concept generates multiple intersections and intermediate spaces from government techniques, ways of negotiating life and diplomatic practices. Biodiplomacy is subsequently used to analyse the pluralistic forms of mediation of estrangement and the negotiation of life that are manifested in liminal spaces. The author argues that biodiplomacy can serve to examine the way in which subjects "negotiate their life" and their "life is negotiated" together and in relation to other multiple organisms and beings.
\end{abstract}

Keywords: Paradiplomacy, biodiplomacy, negotiation of ways of being and living.

\title{
Introducción
}

Los seres humanos, ante las múltiples crisis que ha generado la pandemia del COVID19, andamos buscando nuevas ideas que vuelvan a dar sentido a nuestras vidas. Unas vidas que, en los últimos meses, se han mostrado sumamente frágiles, tanto por la vulnerabilidad que mostramos ante contingencias imprevisibles como por la volatilidad que exhiben las construcciones políticas y sociales que enmarcan y ordenan nuestras formas de ser y vivir. Nos hallamos, así, ante la necesidad de repensar la vida y los modos de existencia.

En este artículo no propondremos una nueva teoría. Tampoco proporcionaremos certezas. Al contrario, trataremos de desdibujar los límites conceptuales de la diplomacia y la paradiplomacia para introducir en el debate el concepto de biodiplomacia. Es al ampliar, extender y, a la vez, plegar los límites de los conceptos cuando se hace posible pensar lo complejo y lo incierto. Además, es en esos pliegues y despliegues donde emergen los conceptos y los fenómenos que anidan en lo liminal. Es decir, en los márgenes, en los espacios intermedios y en los umbrales donde las estructuras establecidas son dislocadas y surgen las incertezas del ser y la inestabilidad de los significados (Malksoo, 2012; Rumelili, 2012; Sorondo, 2019). La(s) diplomacia(s), por ejemplo, entendida(s) como la mediación del extrañamiento o la separación entre entidades, grupos de personas o individuos, solo se hace(n) visible(s) cuando existe una separación o un extrañamiento entre actores.

Ocurre lo mismo con la biodiplomacia. Constantinou y Opondo (2019) argumentan que mediante este concepto se busca superar las limitaciones conceptuales de las visiones tradicionales estatocéntricas de la diplomacia, para hacer emerger múltiples formas diplomáticas que hasta ahora eran consideradas como "privadas", "marginales", o "liminales" (2019, p. 3). De esta forma, y reconociendo que la biodiplomacia opera en y 
a través de múltiples espacios y comunidades epistémicas, sostienen que la "nueva 'realidad' requiere a menudo el reconocimiento y/o la constitución de nuevos sujetos diplomáticos, nuevos interlocutores, nuevas formas de formular una cuestión y métodos de abordarla" (2019, p. 4).

Aunque todavía es pronto para analizar las consecuencias de la pandemia del COVID19, muchos expertos coinciden en que el virus ha actuado como un catalizador, acelerando tendencias económicas, (geo)políticas y sociales preexistentes (Riordan, 2020). La pandemia, por tanto, añade más complejidad a un sistema ya de por sí complejo (Innerarity, 19 de abril de 2020). A este respecto, consideramos pertinente recuperar las primeras líneas del libro Diplomatic Theory of International Relations de Paul Sharp (2009): "Cuando se necesita realizar algo complicado, o cuando se prevé un acuerdo o una mejora general de las relaciones internacionales, a menudo se requiere más y mejor diplomacia" (p. 1). Habría que añadir a la expresión "más y mejor" la fórmula "otras". Es decir, las nuevas realidades políticas, económicas, sociales no solo requieren más y mejor diplomacia, sino también otras formas diplomáticas, menos rígidas y más plurales (Cornago, 2013a).

Este artículo comienza analizando el impacto de la pandemia en la práctica diplomática. Pese a la aceleración de las tendencias recentralizadoras y homogeneizadoras, argumentamos que las prácticas paradiplomáticas o no oficiales siguen siendo relevantes. En el segundo punto nos aproximamos al concepto de paradiplomacia. Una vez relacionado este con los conceptos de gobierno y gubernamentalidad, se intenta tensarlo en el tercer punto. Se busca, así, exponer que la paradiplomacia no es algo que se halle en los márgenes o más allá de los Estados, sino que se encuentra disgregada allá donde se encuentran identidades ambiguas.

En el cuarto punto, en cambio, se introduce el concepto de biodiplomacia. Con esta transición no sostenemos que una vaya a sustituir a la otra. Al contrario, la paradiplomacia sigue siendo el concepto clave para entender la política internacional de las entidades subnacionales. Es más, al revelar las posibilidades de análisis que descubre la biodiplomacia, se advierte que los dos fenómenos exponen una misma realidad; es decir, la pluralización y la naturaleza cada vez más compleja de la diplomacia. A continuación, antes de pasar a las consideraciones finales, se intenta ilustrar lo analizado mediante un ejemplo concreto. En el último punto se presentan algunas opciones analíticas que entreabren el concepto de biodiplomacia.

\section{El impacto del COVID-19 en la diplomacia}

La pandemia del COVID-19 ha sacudido todas las estructuras sociales y políticas. Frente a un virus que no comprende de fronteras ni de territorios nacionales, los Estados, sumidos en el caos y la incertidumbre, e ignorando las múltiples interdependencias e interrelaciones que caracterizan los sistemas sociales, económicos y políticos actuales, comenzaron a reactivar políticas proteccionistas y nacionalistas. El 
cierre de fronteras o las disputas por el material médico son dos ejemplos de políticas y actitudes que se creían olvidadas.

No nos hallamos, sin embargo, ante hechos novedosos. Sabemos que "las crisis aceleran la historia y las tendencias existentes" (Heine, 2020, p. 29). En este sentido, algunos pensadores incluso se atrevieron a afirmar, quizás de manera apresurada, que nos hallamos ante nuevas formas de control de la población. Para Agamben (2020), por ejemplo, estas políticas no serían más que el resultado del uso del "estado de excepción como paradigma normal de gobierno" (p. 18). Estamos, en todo caso, ante la reemergencia, aceleración y vigorización de políticas de control y gestión de las complejas relaciones de fuerza que emergen en lo social.

En el ámbito interno de los Estados, por ejemplo, la concentración de poderes y atribuciones en manos de los gobiernos centrales, en detrimento de la autonomía y capacidad de acción de los gobiernos no centrales, reavivan las políticas centralizadoras. No obstante, como hemos comentado previamente, aún es pronto para sacar conclusiones. Además, aunque los Estados y los gobiernos centrales vuelven a reclamar ser los depositarios de todas las soberanías, son muchos los ejemplos que demuestran que los que actores público-privados de todo tipo siguen manteniendo sus esferas de acción, sus espacios de decisión y sus oportunidades de relación.

Aunque estas formas alternativas políticas queden ensombrecidas por los discursos y las prácticas que enfatizan el carácter unidimensional de los Estados, la realidad se muestra sumamente heterogénea y compleja (REPIT, 2020, octubre 29). A este respecto, la visión descentralizada y plural de la diplomacia tiene la virtud de hacer emerger, tanto la naturaleza heterogénea y compleja de la realidad como las formas múltiples en que la soberanía de los Estados está siendo perforada (Cornago, 2010).

Aunque tradicionalmente la diplomacia ha sido definida como "la conducta de relaciones organizadas entre Estados" (Adler-Nissen, 2016), hoy en día muchas acciones diplomáticas están teniendo lugar en espacios informales donde el Estado, o bien está ausente, o bien está representado de manera indirecta. No nos hallamos ante un fenómeno reciente. En definitiva, tal como señala Cornago (2016).

el origen de la diplomacia se encuentra en la voluntad -y seguramente la necesidad- de los grupos humanos de relacionarse entre sí, de manera estable y pacífica, para vencer el extrañamiento y el asombro original que suscita el descubrimiento de la diferencia y la alteridad. (p. 17).

Sirvan dos ejemplos como ilustración de las múltiples formas de relación e intervención diplomática que han tenido lugar en los últimos meses.

El proyecto europeo S4D4C (Using Science for/in Diplomacy for Addressing Global Challenges) es una iniciativa conformada por individuos particulares, pero que cuenta con el apoyo de universidades e instituciones estatales. Esta plataforma busca fortalecer la diplomacia científica europea y los objetivos de política exterior de la Unión Europea y de sus Estados miembros a través de la ciencia y la cooperación científica, para dar respuesta a los retos globales del futuro. Nos hallaríamos, por tanto, ante relaciones 
"diplomáticas" que se generan con el objetivo de mediar un extrañamiento respecto a unos hechos desconocidos a través del conocimiento.

Otro ejemplo, aunque no tan virtuoso ni ejemplar, lo podemos hallar en el proceso de compra de quinientos respiradores que la Confederación de la Producción y el Comercio de Chile (CPC), como promotor, y el embajador de Chile en China, Luis Schmidt, como coordinador, realizaron en tierras chinas a "revendedores y especuladores". Aunque la participación del embajador de Chile en China parecería dotar de cierta formalidad a esta relación de compra y venta, las condiciones legales y jurídicas en las que se desarrolló dicen lo contrario (Toro, 18 de mayo de 2020).

Estos ejemplos evidencian que la diplomacia se expresa y se representa "en las más diversas expresiones de la vida social" (Cornago, 2010, p. 126). A este respecto, AdlerNissen (2016) argumenta que "el auge de actores no-estatales que van desde compañías transnacionales hasta medios globales, de organizaciones no-gubernamentales a organizaciones multilaterales, cuestionan la imagen de los diplomáticos nacionales como los "custodios de la idea de sociedad internacional"' (p. 100). En el contexto actual, sin embargo, han sido los propios Estados y sus representantes quienes se han mostrado como garantes y, a la vez, elementos disruptivos del orden y de las estructuras normativas que habrían dado vida a aquello que Bull (2002) denominó como sociedad internacional. El robo de material médico, el cierre de fronteras, las acusaciones de incompetencia entre Estados fueron la dinámica general durante los primeros meses de la pandemia. En la actualidad, sin embargo, la carrera por la vacuna y la recuperación económica ha reactivado los espacios de cooperación y colaboración entre naciones, y entre estas y los actores privados.

Nos hallamos, así, ante una pluralización que es reflejo de la transición de la diplomacia desde una arquitectura funcional y simbólica no-controversial y limitada al ámbito institucional y territorial de los Estados hacia un terreno diplomático cada vez más contencioso y sometido a nuevas fuerzas desterritorializadoras y reterritorializadoras (Hardt y Negri, 2000). De esta forma, la visión de la representación diplomática tradicional -que está limitada de manera exclusiva a la representación del Estado, excluyendo de paso múltiples formas de representación alternativas- estaría dejando paso a una visión más plural de la misma.

Las respuestas teóricas a esta ampliación de las agendas y de los actores difieren en cuanto a la aproximación y el objeto de estudio. Constantinou, Cornago y McConnel (2016), por ejemplo, ponen el foco en el proceso de transprofesionalización de la diplomacia. Adler-Nissen (2016), por su lado, señala el fenómeno de la personalización para exponer así las relaciones informales que se dan entre los diplomáticos o entre los diplomáticos y el público extranjero. Cada una de estas aproximaciones, tratando de captar una particularidad concreta, le ha asignado una denominación al fenómeno de la diplomacia. De ahí que se hable de "diplomacia polilateral" (Wiseman, 2004), de "diplomacia catalítica" (Hocking, 1996), de "diplomacia de red" (Metzl, 2001), de "diplomacia transformacional" (Vaisse, 2007), de "diplomacia de participación múltiple" (Susskind, Fuller y Fairman, 2003); de "diplomacia de guerrilla" (Copeland, 
2009), de "megadiplomacia" (Khanna, 2011), de "diplomacia integrativa" (Hocking, Melissen, Riordan y Sharp, 2012) y, por último, de "diplomacia dura" (Sherr, 2013) o "heavy-metal" (Galeotti, 2016).

En este artículo, nos centraremos en el fenómeno de la paradiplomacia para pasar, más tarde, a la biodiplomacia. Ambos conceptos son elusivos. En el caso de la paradiplomacia, a pesar de que está presente en muchas publicaciones, todavía no está del todo definido (Zeraoui, 2016). Merece la pena, por tanto, recuperar la clásica formulación de Butler (cit. en Kuznetsov, 2015), quien en la primera mención que se conoce del concepto empleó la misma para referirse a las diplomacias paralelas y a las personas que complementan o compiten con las formas regulares de acción exterior de los gobiernos centrales.

Aunque en las siguientes secciones defendemos, tal como lo hace Amilhat (2016), la necesidad de "abrir considerablemente el campo de las personas susceptibles de caer dentro de la categoría de 'actores paradiplomáticos"” (p. 57), antes expondremos, de manera breve, los debates que se han dado en torno al concepto de paradiplomacia.

\section{Una aproximación a la paradiplomacia}

La paradiplomacia es un concepto relativamente reciente. Fue en la década de 1980 cuando comenzó a emplearse para reflejar el aumento de la actividad de los gobiernos no centrales. Durante esos años, tal como destaca Keating (2013), la globalización y el ascenso de los regímenes transnacionales suprimió la distinción tradicional entre políticas internas y externas del Estado, permitiendo a los gobiernos subnacionales asumir mayores responsabilidades.

Fueron Duchacek (1990) y Soldatos (1990) quienes elaboraron las primeras tipologías en torno al concepto de paradiplomacia. Desde entonces, muchas terminologías se han empleado para referirse a un mismo fenómeno. Gely (2016) elabora una lista de distintas denominaciones, en la que incluye desde la más genérica, "paradiplomacia", que se emplearía como concepto paraguas (Zeraoui, 2016), hasta "diplomacia de los gobiernos no centrales", "diplomacia multinivel", "diplomacia de las ciudades", "internacionalización de las ciudades", "cooperación descentralizada", o "acción internacional de gobiernos locales". Por su lado, Zeraoui (2011) añade a esta lista los conceptos de "diplomacia paralela", "microdiplomacia", "diplomacia local" o “diplomacia constitutiva" (pp. 62-63).

A este respecto, si bien existe un cierto consenso acerca de las causas que posibilitaron la aparición de la paradiplomacia, se da también un debate mayor sobre lo que debe ser el objeto de estudio. La gran variedad de casuísticas incrementa, además, las ópticas de análisis. De esta forma, hay quienes se centran en analizar las causas que se hallan tras la emergencia del fenómeno (Duchacek, 1990; Kuznetsov, 2015; Lecours, 2002); algunos que examinan los objetivos que se pretenden lograr mediante su práctica (Bernal Meza, 1990; Tussie, 2004); otro autor que amplía su alcance para incluir dentro 
de la paradiplomacia no solo a los gobiernos no centrales, sino a grupos culturales, nacionales o individuales (Senhoras, 2009); y también está quien, directamente, niega validez al concepto como herramienta teórica y de análisis y prefiere emplear otros conceptos (Hocking, 1993a, 1993b).

Sin entrar a examinar los distintos planteamientos, podría decirse de manera general que la paradiplomacia, entendida como la "actividad internacional o la política exterior de las entidades políticas sub-estatales" (Grydehoj, 2014, p. 12), se ha interpretado de dos formas distintas. Mientras hay quien concibe el fenómeno como un subproducto inocente (Duran, 2016) y funcional de la gobernanza multinivel o del sistema político federal, otros lo conciben como una expresión de los antagonismos nacionales y como un espacio de colisión entre entidades subestatales y el Estado central.

Tal como destaca Zeraoui (2016), la primera corriente "hace referencia a una política internacional complementaria entre el Estado central y los gobiernos intermedios" (p. 20). Nos hallaríamos así frente a una diplomacia multicapa o multinivel (Hocking, 1993a), en la que las distintas entidades de gobierno nacionales, subnacionales o de otro tipo y nivel comparten las competencias en política exterior. Dentro de esta corriente se hallarían, por ejemplo, los trabajos que hablan de diplomacia local (Dávila, Schiavon y Velázquez, 2008), federativa (Schiavon, 2010) o constitutiva (Kincard, 2009).

La segunda corriente, en cambio, destaca las disputas competenciales y simbólicas que se generan entre los distintos gobiernos cuando se trata de cuestiones como la representación política y diplomática. La paradiplomacia reflejaría así "la capacidad de política exterior de gobiernos sub-nacionales, regionales o locales" (McConnell, Moreau y Dittmer, 2012, p. 805). Estas capacidades, a su vez, están ligadas al poder de hacer y de no hacer. O, como dice una cita de Aristóteles, "donde en nuestra mano está el hacerlas, está también el dejarlas de hacer” (Simón, 2001, p. 61). Es coherente pensar, por tanto, que algunas veces los intereses de las distintas entidades coincidirán y las capacidades convergerán, mientras que otras veces los intereses se opondrán y las distintas entidades emplearán sus capacidades para alcanzar sus propios intereses particulares, dándose así múltiples conflictos. En estos casos, se suele emplear los conceptos de paradiplomacia identitaria o protodiplomacia (Paquin, 2004).

Por el contrario, muchos otros planteamientos navegan entre ambas corrientes y se desplazan entre conceptos opuestos, reflejo de la complejidad de la política. Sea cual sea la lectura, la paradiplomacia muestra, sobre todo, la existencia de nuevas formas diplomáticas y, consecuentemente, de nuevas formas de extrañamiento. Extrañamientos nuevos que poco tienen que ver con los antiguos extrañamientos entre Estados que la diplomacia tradicional decía mediar. Ahora hablamos de extrañamientos que emergen dentro de los Estados, pero que responden a procesos que se dan tanto en el interior como en el exterior de los límites territoriales estatales. Estas son dinámicas que al cuestionar la homogeneidad de los Estados ponen en duda, a su vez, todo el sistema intraestatal. Ello no quiere decir, sin embargo, que nos hallemos ante el desmembramiento del Estado, ni ante la desaparición de las formas tradicionales de la diplomacia. Estaríamos, en todo caso, ante aquello que McConnell, Moreau y Dittmer 
(2012) denominan como "ensamblaje diplomático" (p. 812). Es decir, un haz complejo de relaciones en las que, paradójicamente, estas formas paradiplomáticas o "no oficiales" refuerzan, en muchos casos, la hegemonía de las "formas oficiales" de diplomacia.

Esta paradoja se hace visible, por ejemplo, en el doble deseo que expresan los discursos paradiplomáticos. Por un lado, estas formas "no oficiales" manifiestan el deseo de ser reconocidas como actores de pleno derecho en la arena internacional. Por otro lado, en cambio, el deseo de ser reconocidas como iguales suele ir acompañado de un "deseo distintivo de autonomía política" (Cornago, 2013b, noviembre, p. 3). Este doble deseo muestra que, todavía hoy, el "marco diplomático" estatocéntrico tradicional es el "estándar de oro" al que aspira la mayoría de las formas nuevas o no oficiales de la diplomacia. Hay, por supuesto, alguna que otra excepción: la herramienta teóricametodológica de la transdiplomacia propuesta por Arévalo (2017), que busca "investigar fenómenos diplomáticos desde una perspectiva holística, dinámica, flexible, abierta, crítica, provocativa e hipotética de la historia” (2017, p. 144); o la práctica teórica de la diplomacia desde debajo de Ghilarducci (2020), que "permite experimentar una reapropiación entre ciertos márgenes, de un protagonismo directo en el campo de las relaciones internacionales por parte de sujetos políticos colectivos no estatales" ( $p$. 121), son algunas mociones que se resisten a imitar ese "estándar de oro".

En definitiva, cuando hablamos de paradiplomacia estamos ante un fenómeno ambivalente. Para seguir despejando sus múltiples interpretaciones y sentidos y, a la vez, comenzar a introducir el concepto de biodiplomacia, trataremos de responder a una cuestión formulada por Aguirre (2013) en su intento por comprender la paradiplomacia. Este, tras afirmar que la idea de gobierno es clave para comprender la paradiplomacia, plantea la siguiente pregunta: ¿qué queremos decir realmente cuando hablamos de gobierno en la política contemporánea, tanto nacional como extranjera?

\section{Ampliando el alcance de la paradiplomacia a través de la idea de gubernamentalidad}

Si bien la pregunta de Aguirre es pertinente y acierta a la hora de afirmar que en la actualidad 'el clásico 'monismo' antropomórfico del estado ha sido sustituido, definitivamente, por una comprensión mucho más sofisticada de la naturaleza compleja, continuamente superpuesta y esencialmente 'pluralista' de los sistemas políticos contemporáneos” (2013, p. 199, traducción mía), su respuesta no llega a captar la enorme potencialidad que tiene un concepto que él mismo emplea en el artículo. Hablo de la gubernamentalidad. Aguirre usa este concepto para argumentar dos cosas: por un lado, que los actores gubernamentales internacionales autónomos no centrales "son representaciones territorializadas y 'localizadas' de un estado constitucionalmente "plurinacional"'; y, por otro lado, a nivel puramente fáctico y político, "que la 'gubernamentalidad' significa que estos gobiernos no centrales están a cargo de la 
administración pública en un ámbito muy amplio de competencias legislativas constitucionales 'exclusivas"' (Aguirre, 2013, pp. 203-204; traducción mía).

La gubernamentalidad, sin embargo, no es solo relevante para comprender la distribución de competencias o el nivel de representación de los distintos gobiernos en el ámbito internacional. El propio Aguirre (2013) argumenta que la gubernamentalidad de los actores no centrales autónomos es relevante para comprender la paradiplomacia. Añade, además, que

la falta de una interpretación teórica sólida de las diversas formas de 'gubernamentalidad' de hoy en día puede servir para explicar las numerosas contradicciones semánticas, ideológicas y constitucionales que se extienden por la literatura acerca de la participación internacional de los gobiernos no centrales. (2013, p.198, traducción mía).

Invirtiendo su frase se podría argumentar que una mayor comprensión de la naturaleza compleja de la gubernamentalidad podría servir para explicar las múltiples formas de diplomacia o mediación del extrañamiento.

A este respecto, a pesar de que cuando hablamos del gobierno o de formas de gobernar, la mayoría de las veces establecemos una relación de continuidad con el Estado, Foucault (2016) lanzó una advertencia al percatarse de que el gobierno es algo más que una prerrogativa del Estado. A partir de esta primera aclaración, la gubernamentalidad pasó a concebirse como una racionalidad específica que habría hecho aparición en la Europa occidental durante la modernidad temprana, como resultado de un arte de gobierno particular del Estado en el que técnicas y conocimientos de las ciencias sociales y humanas fueron incorporadas a la tarea de gobierno (Dean, 2010). La gubernamentalidad, por tanto, es algo más que unas simples competencias en el ámbito de la administración pública y algo más que una mera representación de un Estado plurinacional.

La gubernamentalidad expone las múltiples formas de poder constituidos por una "variedad de tácticas, estrategias, espacios de verdad y racionalización" (Makarychev y Yatsyk, 2016, p. 45). Formas de poder y arte de gobierno que se emplean para "racionalizar los problemas planteados a la práctica gubernamental por los fenómenos propios característicos de un conjunto de seres vivos constituidos como población" (Foucault, 2016, p. 311). Foucault dio el nombre de biopolítica a estas formas de poder y a este arte de gobierno. La biopolítica se trata, por tanto, del perfeccionamiento del arte de gobierno a través de la "administración, orquestación, producción y reproducción de las poblaciones y la vida -donde la promoción de vida, más que el poder de dar muerte, se convierte en el objeto central y propósito de poder" (Selby, 2007, p. 333). Un arte que se emplea para controlar las relaciones "entre la raza humana, o los seres humanos en tanto que son seres vivos, y su entorno, el ámbito en el que viven" (Foucault, 2003, p. 247). Parece claro, por tanto, que el arte de gobernar, en la actualidad, no puede concebirse como una atribución exclusiva del Estado. 
Una de las características principales de la biopolítica es que se desarticula en un "conjunto de poderes administrativos que están, de alguna forma, fuera de los aparatos del propio Estado" (Butler, 2004, p. 55). Sin embargo, esta pérdida de soberanía se compensa, a su vez, "a través del resurgimiento de la soberanía dentro del espacio de la gubernamentalidad" (2004, p. 56).

Esta desarticulación del gobierno y el poder influye, sobremanera, en el modo de comprender la naturaleza de la paradiplomacia. Constantinou y Opondo (2019) afirman, por ejemplo, que "las implicaciones más amplias del fenómeno diplomático se ponen de manifiesto cuando se considera la forma en que esta se entrecruza con nuestra comprensión de la biopolítica e influye en ella" (p. 3). Ocurre lo mismo si invertimos la ecuación. Es decir, una mayor comprensión de las formas actuales de gubernamentalidad nos facilitará la interpretación de las nuevas formas diplomáticas.

En este sentido, la paradiplomacia, tal como destaca Duran (2016), "en virtud de su peso político y gubernamental, así como por el lugar que ocupa en el complejo entorno diplomático multi-nivel característico de nuestro actual panorama internacional" (p. 5) puede ser una herramienta o concepto útil para analizar los extrañamientos actuales. Todavía hoy, como consecuencia del gran abanico de posibilidades que entreabre para expandir los estudios internacionales e incorporar al actor subestatal (Álvarez, 2017), la paradiplomacia sigue siendo un concepto clave para comprender las dinámicas internacionales.

Al inicio del artículo hemos argumentado que la diplomacia emerge en los espacios intermedios o liminales, cuando se hace necesario mediar las formas diversas de extrañamiento. Stengers (2011) afirma, al respecto, que la diplomacia, como una especie de desidentificación, se convierte en una "experiencia de paso"; una "técnica que navega 'la tensión entre la territorialidad y la desterritorialización'” (2011, pp. 376377). Duran (2016), por su lado, afirma que la paradiplomacia puede ser concebida "como un espacio específico de mediación diplomática, situada en un punto intermedio entre el juego de poder 'realista' y la necesidad humanista de concretarse y comprometerse con los demás” (p. 5).

Es, precisamente, para comprender y alumbrar las múltiples intersecciones y los espacios intermedios que se generan entre técnicas de gobierno, formas de negociar la vida y prácticas diplomáticas, que Constantinou y Opondo (2019) emplean el concepto de biodiplomacia. Un concepto y una tarea que, tal como argumentan, "puede ser productiva para imaginar la vida y los modos de co-existencia dentro y más allá del entorno gubernamental" (Constantinou y Opondo, 2019, p. 2).

Parecería, así, que cuando hablamos de paradiplomacia y de biodiplomacia estaríamos hablando de lo mismo. No obstante, existe una diferencia relevante entre ambos conceptos. Mientras que la paradiplomacia permite analizar, tanto la forma de mediación o ampliación de los extrañamientos que emergen entre actores y sujetos, como la forma en que esos mismos actores y sujetos actúan y desarrollan relaciones en los ámbitos internos y/o externos de los Estados, la biodiplomacia hace visible la forma 
en que la vida de esos actores y sujetos es negociada. Ambos tienen, empero, una cosa en común: son el resultado del carácter plural y complejo de la diplomacia.

\section{Sobre la biodiplomacia y las formas de ser y vivir}

Constantinou y Opondo (2019) definen la biodiplomacia como "la continua negociación de los significados y la materialidad de determinadas formas de vida frente a otras formas de ser" (p. 1). Es decir, frente a los procesos y prácticas de conducta, gobierno y optimización activadas por regímenes gubernamentales de todo tipo que operan en red, y actúan entre y a través de las fronteras y las poblaciones territoriales, la biodiplomacia expondría otras formas de vivir, ser y negociar la vida; concretamente, aquellas formas que se opondrían a la expansión de las formas biopolíticas.

Sin embargo, el concepto de biodiplomacia no es nuevo. A pesar de ello, no existe un consenso acerca de su significado, ni tampoco acerca de los objetivos que debe seguir o acerca de los retos a los que debe responder (Aguilar y Paterman, 2020). Como consecuencia de esta indefinición, el concepto se ha empleado para analizar los procesos de gestión y protección del medioambiente o la biodiversidad; las negociaciones en torno a la conservación y uso responsable de los recursos naturales (Sánchez y Juma, 1994); o las nuevas tecnologías de producción, reproducción y gestión de la vida en campos como la biotecnología o la bioética (Calestous, 2005), entre otras cuestiones. Tal como señalan Aguilar y Paterman (2020), a pesar de que no existe un sentido unificado, estas aproximaciones consideran que la "biodiplomacia es parte de la diplomacia clásica, solo que consideran la necesidad de adoptar un enfoque global e integrado para la gestión de los desafíos mundiales que afectan a la biosfera" (pp. 23-24).

Sin restarle relevancia a las políticas medioambientales o a las "artes de preservación y promoción de vidas mediante toda forma de cooperación internacional entre estados" (Vlavianos-Arvanitis, 2005), Constantinou y Opondo (2019) tratan de ampliar el alcance del concepto para superar las limitaciones que generan las interpretaciones convencionales de la diplomacia. Previamente, sin embargo, fue Konrad (2007) quien había planteado una aproximación ética y antropológica al concepto de biodiplomacia, marcando el camino que seguirían años más tarde Constantinou y Opondo. Konrad (2007) emplea la biodiplomacia para analizar las relaciones biodiplomáticas en las ciencias internacionales: las modalidades de intervención, formas de acción y desconexión en el ámbito biodiplomático, y el funcionamiento de "espacios de transacción" entre prácticas de intergobernabilidad e interdisciplinaridad. De esta forma, se aleja de los “análisis de 'biopoder' inspirados por Foucault” (Konrad, 2007, p. 329) y hace emerger las prácticas denominadas como biodiplomáticas.

Siguiendo la senda de Konrad, Constantinou y Opondo (2016) argumentan que la biodiplomacia permite analizar los procesos de negociación de la vida que acompañan a la expansión global de "las estrategias de control, discursos de legitimación y formas de 
cooptación y cohabitación más allá de la gobernanza" (p. 309). Es decir, permite explorar los procesos que van aparejados a las formas biopolíticas y argumentar que las vidas no son ya simplemente gobernadas, sino que también son negociadas, haciendo que algunas sean admisibles y otras inadmisibles.

Aunque el planteamiento de Constantinou y Opondo (2016) tenga la virtud de exponer las contraconductas y las formas alternativas de vivir que operan más allá de las prácticas gubernamentales y más allá de la conducta de las conductas, la ruptura ontológica que establecen entre la biopolítica y la biodiplomacia no es tan clara; o, por lo menos, puede ser problematizada. Al problematizarlo, sin embargo, se advierte que el concepto no pierde relevancia. Al contrario, alcanza una nueva dimensión, pues permite analizar tanto las prácticas biopolíticas como las prácticas y las formas de negociar la vida que se oponen o se ejercen en los márgenes de la biopolítica. Además, en el contexto actual, cuestiones como la protección de los ecosistemas, el amparo de "comunidades vulnerables cuyas vidas pueden ser afectadas por determinados riesgos sanitarios, medioambientales o científicos" o, en general, el cuidado de "ciertas prácticas que implican movimientos transnacionales a través de las fronteras" (Konrad, 2007, p. 342) reclaman nuestra atención más que nunca.

En este sentido, la biodiplomacia puede emplearse de tres formas que se interrelacionan entre sí. Por un lado, tal como lo hacen Constantinou y Opondo $(2016,2019)$ para analizar la forma en que los sujetos "negocian su vida". Pero también puede emplearse en sentido contrario; es decir, para comprender la manera en que la biopolítica, en la actualidad, se extiende hacia amplios espacios sociales y hacia el ámbito privado del individuo. De esta forma, la vida de los sujetos pasa a estar negociada. La biodiplomacia, por tanto, es un concepto que visibiliza las dos caras de una misma moneda. Tal como señala Goikoetxea (2017), a menudo las capacidades y técnicas de poder que "modulan y domestican nuestros cuerpos individuales y colectivos, y las que nos hacen iguales y libres" (p. 17) son de la misma tipología.

Este doble sentido de la biodiplomacia puede ilustrarse analizando el término diplomacia. Etimológicamente, la diplomacia se compone del vocablo fí $\lambda \lambda$ o (diplo), doblar en dos, y del sufijo $\mu \alpha$ (ma), que hace alusión a un objeto. Cambiando el objeto por el sujeto, podríamos argumentar que la diplomacia "dobla al sujeto" en dos; bien porque hace doblar la conciencia de los sujetos (en dos), bien porque enmarca las condiciones de posibilidad, la episteme o el marco del saber del mundo (en dos). Tal como argumentase Foucault (2003), la biopolítica es la política que se hace responsable de la administración de la vida, controlando las relaciones entre los seres humanos "en tanto que son seres vivientes, y su entorno, el ámbito en el que viven” (2003, p. 247).

En este sentido, aunque la interrelación entre la diplomacia y la gubernamentalidad no sea explícita, es relevante destacar que esta opera en lo imaginario, lo simbólico y lo funcional. En cuanto al nivel de lo imaginario, Death (2011), haciendo referencia a las representaciones que en las cumbres diplomáticas tienen lugar, afirma que estas pueden comprenderse "desde una perspectiva gubernamental, como una forma de poder a distancia, mediante el que los patrones de conducta son dirigidos por horizontes de 
inteligibilidad discursiva" (p. 6). El propio Constantinou (1996), por su lado, resalta los imaginarios que genera la diplomacia como proceso de enmarcamiento. De manera similar, Banai (2014) afirma que la diplomacia prescribe de manera normativa el imaginario público. Imaginarios que se piensan, se hacen visibles y hacen ver el mundo de una determinada forma.

Respecto al nivel simbólico y funcional, al relacionar ambos niveles, Stetter (2016) sostiene que mientras que antaño la diplomacia se sustentaba sobre su rol o fuerza simbólica, que se erigía sobre la estética de lo sublime, en la actualidad se sustenta sobre su funcionalidad práctica, que pasa por promover los bienes globales interminablemente. De esta forma, hoy en día, su estética de lo sublime depende de su carácter irremediable, puesto que el mundo emerge como un espacio a gobernar y la diplomacia se representa como "la piedra angular de la gubernamentalidad" (2016, p. 394).

La palabra diplomacia, sin embargo, puede adquirir también otro sentido empleando la misma carta etimológica. Haciendo uso del doble sentido del concepto de "doblar", Constantinou (1996) expone las formas dobles y economías de la diplomacia. Formas dobles no oficiales que, como en el caso del reinado de Luis XV, se llevaban a cabo en paralelo con las oficiales. Diplomacias dobles que eran "oficialmente no oficiales y no oficialmente oficiales" (Constantinou, 1996, p. 85). Un doble extraoficial que, precisamente, por su carácter no oficial puede operar fuera o en los márgenes de los límites legales y normativos definidos por el "marco diplomático" estatocéntrico tradicional. Formas extraoficiales que, en muchos casos, pueden transcender las formas y modos oficiales; y formas dobles extraoficiales que pueden ser empleadas, a menudo, más veces y de más maneras que aquellas formas oficiales que doblan.

De cierta manera, según Constantinou y Opondo (2019), el concepto de biodiplomacia no es más que la materialización de estas dobles formas diplomáticas. Es decir, formas de negociación de la vida y formas plurales de existencia que permiten vislumbrar los "límites de la biopolítica, mientras se generan otros dominios de relación" (p. 11) que transcienden los límites territoriales del Estado. Ante los procesos de conducta, gobierno y optimización activados por regímenes gubernamentales de todo tipo que operan en red, y que actúan entre y a través de fronteras y poblaciones territoriales, la biodiplomacia señala las formas de negociación de la vida que se oponen, dentro de lo posible, a estas formas de biopolítica nacionales $y$ transnacionales. Esta conceptualización se asemeja a la idea de multitud de Hardt y Negri (2000), concepto de multitud que, tal como señala Lemke (2017), designa "la totalidad heterogénea y creadora de actores que se mueven en la inmanencia de las relaciones de poder sin remitirse a una instancia de mayor importancia o a una identidad subyacente" (p. 93).

Vemos, por tanto, que la biodiplomacia permite analizar la forma en que los sujetos "negocian su vida" y, a la vez, permite comprender la manera en que la biopolítica se desarticula en múltiples espacios sociales e individuales, haciendo que la vida y la conducta de estos sujetos "sea negociada" y conducida. 
Por último, nos queda la tercera de las formas de comprensión de la biodiplomacia. Aquella que, situando la vida en el centro de los discursos y de las prácticas políticas, destaca las múltiples relaciones y formas de coexistencia y conflicto que se generan en el planeta Tierra. Es decir, en el ecosistema global del planeta Tierra donde los seres humanos no somos más que unos organismos, seres o sujetos vivos que debemos negociar nuestra vida, o nuestra vida puede ser negociada junto y en relación con otros múltiples organismos y seres que conforman el ecosistema global. En la actualidad, en lo que se viene llamando como la era del Antropoceno, los seres humanos no podemos darle la espalda al entorno en el que vivimos, ni tampoco a los otros seres vivos y no vivos con los que cohabitamos. No podemos mostrarnos ajenos a la profundamente interdependiente biosfera.

En este sentido, Constantinou y Opondo (2019) destacan que el concepto de biodiplomacia nos incita a "pensar sobre relaciones de vida/no vida en formas que exceden las bio-ontologías" (p. 13). Además, formulan, no sin cierto reparo, una propuesta de carácter normativo con la que destacan la relevancia de la biodiplomacia como "práctica política que subscribe y ayuda a la 'pluriversalidad' de ontologías, modos de existencia, mundos vitales, proyectos políticos, y cosmologías que la universalidad occidental pensaba haber erradicado" (2019, p. 17). Prácticas políticas que promueven "versiones cosmopolitas más inclusivas y post-fundacionales, que expanden el cosmos y aprecian mejor los modos plurales de existencia" (2019, p. 17).

Si algo nos ha mostrado la pandemia del COVID-19 han sido nuestros propios límites. Dussel (cit. en Pérez Pirela, 7 de agosto de 2020) afirma, al respecto, que la naturaleza se ha levantado contra el ser humano para hackear, de esta manera, el "proyecto" de la modernidad. Las promesas de la ciencia y la técnica ya no mitigan la inseguridad ontológica y la desconfianza que los seres humanos sienten ante una autoidentidad inestable y ante los cambios constantes en los entornos sociales o materiales en los que deben desarrollar su vida. El "intento fáustico de someter la vida entera al control absoluto del hombre bajo la guía del conocimiento" (Castro-Gómez, 2000, p. 88) se asemeja, cada vez más, al mito de Sísifo. Cuando creemos estar cerca de controlar la naturaleza, esta se revuelve y nos muestra que las prácticas racionales que nos han ido acercando a la cima de la montaña no tomaban en consideración e, incluso, ignoraban aquello que posibilitaba nuestra ascensión; ergo, la misma existencia de la naturaleza y de la montaña. Si la idea de la modernidad nos prometía superar el extrañamiento que nos produce lo desconocido, la pandemia nos ha mostrado que los seres humanos no solo debemos mediar los múltiples extrañamientos que emergen entre nosotros, sino que nuestra relación con la naturaleza -y con otros seres vivos/no vivos- está atravesada, también, por múltiples otros extrañamientos. La biodiplomacia, al emplazar la vida en el centro y al entrar "en conversación con la negociación de modos de existencia" (Constantinou y Opondo, 2019, p. 13), engarza los múltiples extrañamientos con las múltiples mediaciones que pueden desarrollarse mediante formas plurales de diplomacia.

Antes de pasar a las consideraciones finales, en el siguiente punto trataremos de ilustrar, mediante un ejemplo concreto, las múltiples interrelaciones que se dan en la vida real 
entre los tres planos que hemos ido desarrollando a lo largo del artículo. Es decir, entre las formas paradiplomáticas o formas plurales no oficiales de diplomacia, las formas biodiplomáticas de negociar la vida y las formas de gubernamentalidad y biopolítica de control y gestión de la vida. Todo ello, en el contexto de una pandemia que hace visible la fragilidad de nuestras vidas y nuestra dependencia del entorno natural en el que vivimos.

\section{Una ilustración: venezolanos afuera de su embajada en Santiago de Chile}

El cierre de fronteras y las restricciones en el transporte aéreo y terrestre que se produjeron como consecuencia del COVID-19 dejó varado a muchos migrantes y turistas alrededor del mundo. Es el caso, por ejemplo, de cientos de venezolanos que, hallándose en Chile, se vieron ante la necesidad de volver a Venezuela por las consecuencias económicas que derivó la pandemia (Aravena, 19 de junio de 2020). La crisis sanitaria, social y económica desatada por el coronavirus, y la urgencia por volver, pero la imposibilidad de hacerlo, obligó a muchos de ellos a acampar en las afueras de la Embajada de Venezuela, situada en la comuna de Providencia, en Santiago. Este problema no era exclusivo de los venezolanos. Colonias de peruanos y bolivianos hicieron lo mismo frente a sus respectivas representaciones diplomáticas. Otras múltiples colonias, en cambio, aquellas que poseían mayores recursos, esperaron en sus respectivas viviendas hasta que, mediante vuelos humanitarios, al principio, y vuelos regulares, después, pudieron volver a sus países de origen.

Expondremos el caso de los venezolanos, puesto que ilustra a la perfección las prácticas plurales oficiales y no oficiales de diplomacia que venimos destacando a lo largo del trabajo; ya sea en forma de paradiplomacia y mediación del extrañamiento, o ya sea en forma de biodiplomacia y negociación de la vida.

Aunque en agosto de 2018 el Gobierno de Venezuela puso en marcha el Plan de Vuelta a la Patria, que "establece un puente aéreo y terrestre para el retorno voluntario de todos aquellos migrantes y sus familias que carezcan de medios propios para el regreso" (Gobierno Bolivariano de Venezuela, 2020), y aunque la Convención de Viena de Relaciones Consulares introdujo dentro de la ley internacional los derechos y las responsabilidades concernientes a la protección de connacionales, la Embajada de Venezuela en Chile apenas respondió a las peticiones iniciales. Mientras que el Gobierno venezolano, como todos los otros gobiernos, se vio superado por la pandemia, para el Gobierno central de Chile este problema era menor. Paradójicamente, la puerta de la Embajada de Venezuela se convirtió, así, en una frontera interna. Una frontera que emplazaba a los venezolanos allí acampados entre la Embajada, como territorio jurídicamente venezolano, y la calle o la tienda de campaña en el que dormían durante las noches, como espacio que, por entonces, era jurídicamente ilegal, pues en la capital regía el toque de queda y la llamada al confinamiento.

Frente a esta situación, fueron las comunas de Santiago y sujetos individuales los que se movilizaron para encontrar soluciones o para paliar la situación de precariedad y 
vulnerabilidad en la que se encontraban los ciudadanos venezolanos. Mientras que las comunas, en colaboración con instituciones privadas como el Servicio Jesuita de Migrantes, habilitaron u ofrecieron espacios para que estas personas pudiesen pasar la noche bajo un techo (Villarroel, 27 de mayo de 2020), otras personas, a título individual, se acercaron a las afueras de la Embajada a ofrecer ayuda en modo de alimento, mantas u otros víveres. Fueron, por tanto, los gobiernos no centrales, pero también los actores individuales quienes mediante formas paradiplomáticas llenaron el vacío dejado por los gobiernos centrales o las instituciones estatales, mostrando así las distintas estructuras y formas de acción y comunicación que diferencian a la diplomacia desde debajo de la diplomacia oficial (Ghilarducci, 2020).

Aunque el ejemplo que estamos exponiendo requiere de un análisis más profundo para comprender las diversas realidades y problemáticas que se ocultan tras las imágenes tan impactantes que dejaron, por ejemplo, los niños que en plena pandemia y bajo un clima invernal se hallaban acampados en las afueras de una embajada, sí permite mostrar, por un lado, que el deber de cuidado, en la actualidad, ha sufrido una inversión como resultado de una racionalidad política que concibe a los seres humanos como un "objeto de protección, pero también como un recurso de movilización" (Adler-Nissen y Tsinovoi, 2018, p. 211). Por otro lado, expone los límites de la diplomacia tradicional estatocéntrica y la emergencia de otras formas diplomáticas. De este modo, frente a las formas biopolíticas de negociar la vida que exhortan a los seres humanos a ser "proveedores activos de su propia protección" (2018, p. 213) a través de la contratación de seguros privados $\mathrm{u}$ otros productos mercantiles si no quieren verse abandonados frente a acontecimientos azarosos, fuerzas naturales o crisis sistémicas, las relaciones de cuidado, protección y solidaridad que se desarrollaron entre las personas acampadas, y entre estas y las personas que se acercaron al campamento para ofrecer ayuda desinteresada, exhiben otras formas de negociar la vida y la existencia.

\section{Consideraciones finales}

Se ha afirmado al inicio de este artículo que no era nuestro propósito proponer teorías ni proporcionar certezas. Al contrario, se ha tratado de tensar los límites epistemológicos de conceptos como la diplomacia o la paradiplomacia para extender los horizontes teóricos, introducir el concepto de biodiplomacia en el debate y explorar los espacios liminales en los que emergen las formas plurales de mediación de extrañamiento y negociación de la vida.

El análisis de los márgenes y los puntos intermedios permite, además, establecer relaciones entre distintos fenómenos y analizarlos a partir de nuevos conceptos y ejercicios teóricos. El concepto de biodiplomacia, por ejemplo, en el contexto actual puede emplearse para analizar tres cuestiones de gran relevancia. Por un lado, tal como se ha hecho en este artículo, para exponer las formas plurales de diplomacia que se (re)producen o toman forma en innumerables relaciones diarias y cotidianas. De esta forma, la diplomacia se desliga del Estado y recupera su sentido original; que no es otro 
que el de mediar las relaciones con los otros ajenos y tratar de adquirir conocimiento de aquello que nos es extraño (Der Derian, 1987).

Constantinou (2006) denominó homodiplomacia a estas formas diplomáticas que responden a la necesidad que tenemos los seres humanos de repensar las formas de relación que desarrollamos entre nosotros, y entre nosotros y el entorno que habitamos. Emulando a Ghilarducci (2020, p. 132) y su "paradiplomacia homodiplomática", se podría hablar de una biodiplomacia homodiplomática; es decir, una forma de "conocimiento de uno mismo -y crucialmente este conocimiento del uno mismo como un modo más reflexivo de abordar y transformar las relaciones con los demás" (Constantinou, 2006, p. 352), así como con el entorno que habitamos. En definitiva, la forma en que negociamos nuestra vida.

Por otro lado, puede emplearse para pensar las formas de producción y reproducción de la subjetividad humana. Hemos afirmado anteriormente que la biodiplomacia, al igual que la diplomacia, emerge en los espacios intermedios o liminales. En el contexto actual, donde la incertidumbre acentúa la "experiencia fronteriza" en que se (re)produce la subjetividad (Mendiola, 2001), merece la pena analizar las formas en que la vida de los sujetos puede ser negociada/controlada por otros (Esposito, 28 febrero de 2020).

Por último, en concordancia con el punto anterior, la biodiplomacia permite reflexionar sobre el fenómeno de las relaciones transfronterizas. Para ello, no obstante, las fronteras no deben concebirse, únicamente, como delimitaciones territoriales, sino como "efectos" (Mendiola, 2001, p. 205) o como "dispositivos complejos y móviles" (Amilhat, 2016, p. 50) que se encuentran "dispersados un poco por todas partes, en dondequiera que el movimiento de la información, personas y cosas está dándose y controlándose" (Balibar, 2004, p. 1). Es decir, límites que, aunque tengan su concomitante territorial, son ante todo sociales (Sánchez, 2014). En el contexto actual, por ejemplo, cabría preguntarse de qué forma se articula, se desarrolla y se practica la biodiplomacia cuando nos hallamos confinados en casa, cuando la puerta del domicilio comienza a asemejarse a una frontera infranqueable que nos separa de la naturaleza (Aponte y Kramsch, 2020).

Vemos, por tanto, que la biodiplomacia posibilita el análisis reflexivo. Tarea que, en la actualidad, cuando el control de la pandemia parece exigir la restricción y la limitación de derechos, se hace más imperante que nunca. Solo así podremos concebir formas de vida y existencia que valgan la pena vivir cuando llegue la tan ansiada "normalidad".

\section{Referencias bibliográfícas}

Adler-Nissen, R. (2016). Diplomatic agency. En C. M. Constantinou, M. Keer y P. Sharp (eds.), The Sage Handbook of Diplomacy (pp. 92-103). Londres: SAGE.

Adler-Nissen, R. y Tsinovoi, A. (2018). Inversion of the "duty of care": diplomacy and the protection of citizens abroad, from pastoral care to neoliberal 
governmentality. The Hague Journal of Diplomacy, 13(2), 211-232. DOI: 10.1163/1871191X-11302017

Agamben, G. (2020). La invención de una pandemia. En P. Amadeo (ed.), Sopa de Wuhan (pp. 17-19). Editorial ASPO. Recuperado de https://www.elextremosur.com/files/content/23/23684/sopa-de-wuhan.pdf

Aguilar, A. y Paterman, A. (2020). Biodiplomacy, the new frontier for bioeconomy. New Biotechnology, 59, 20-25. Recuperado de https://doi.org/10.1016/ j.nbt.2020.07.001

Aguirre, I. (2013). Making sense of paradiplomacy? An intertextual enquiry about a concept in search of a definition. En F. Aldecoa y M. Keating (eds.), Paradiplomacy in Action: The Foreign Relations of Subnational Governments (pp. 185-209). Nueva York: Routledge.

Álvarez, M. (2017). El rol de la paradiplomacia en las entidades binacionales: análisis del accionar de las provincias argentinas y regiones chilenas en los casos de EBITAN y EBIFETRA. Si Somos Americanos. Revista de Estudios Transfronterizos, 17(2), 77-95. Recuperado de http://dx.doi.org/10.4067/S071909482017000200077

Amilhat, A-L. (2016). Gentes y agentes, condiciones paradiplomáticas de la creación de una frontera móvil. En. S. González, N. Cornago y C. Ovando (eds.), Relaciones transfronterizas y paradiplomacia en America Latina (pp. 47-71). Santiago: RIL Editores.

Aponte, J. y Kramsch, O. (2020). Las fronteras de la COVID-19: ¿escenario de guerra o camino de esperanza? Un diálogo telemático transatlántico. Geopolítica(s). Revista de Estudios sobre Espacio y Poder, 11 (Especial), 39-51. Recuperado de https://doi.org/10.5209/geop.69129

Aravena, A. (19 de junio de 2020). "Vuelta a la Patria"... venezolanos a la deriva en las puertas de su embajada. Biobiochile.cl [en línea]. Recuperado de https://www.biobiochile.cl/noticias/opinion/tu-voz/2020/06/19/vuelta-la-patriavenezolanos-la-deriva-las-puertas-embajada.shtml

Arévalo, G. A. (2017). La diplomacia indígena: un enfoque transdiplomático. Si Somos Americanos. Revista de Estudios Transfronterizos, 17(1), 141-169. Recuperado de http://dx.doi.org/10.4067/S0719-09482017000100141

Balibar, E. (2004). We, The People of Europe? Princeton: Princeton University Press.

Banai, H. (2014). Diplomatic imaginations: mediating strangement in world society. Cambridge Review of International Affairs, 27(3), 459-474. Recuperado de https://doi.org/10.1080/09557571.2012.744640

Bernal Meza, R. (1990). El papel de las regiones en la formulación de la política exterior y potencial de articulación con regiones de países limítrofes. 
Antecedentes y perspectivas. Integración Latinoamericana, 15(156), 28-39. Recuperado de https://www.jstor.org/stable/41391318

Bull, H. (2002). The Anarchical Society (3ª ed.). Londres: Palgrave.

Butler, J. (2004). Precarious Life. Londres: Verso.

Calestous, J., (2005). The New Age of biodiplomacy. Georgetown Journal of International Affairs, 6(1), 105-114. Recuperado de https://www.jstor.org/ stable/43134079

Castro-Gómez, S. (2000). Ciencias sociales, violencia epistémica y el problema de la invención del otro. En Clacso, La colonialidad del saber: eurocentrismo y ciencias sociales. Perspectivas latinoamericanas (pp. 88-98). Buenos Aires: Consejo Latinoamericano de Ciencias Sociales (Clacso).

Constantinou, C. M. (1996). On the way to Diplomacy. Minneapolis: University of Minnesota Press.

Constantinou C. M. (2006), “On Homo-Diplomacy”. Space and culture, 9(4), 351-364. DOI: https://doi.org/10.1177/1206331206290135

Constantinou, C. M. y Opondo, S. O. (2016). Engaging the 'ungoverned': The merging of diplomacy, defence and development, Cooperation and Conflict, 51(3), 307324. DOI: https://doi.org/10.1177/0010836715612848

Constantinou, C. M. y Opondo, S. O. (2019). On biodiplomacy: Negotiating life and plural modes of existence. Journal of International Political Theory, octubre, 121. DOI: $10.1177 / 1755088219877423$

Constantinou, C. M., Cornago, N. y McConnel, F. (2016). Transprofessional diplomacy. Diplomacy and Foreign Policy, 1(4), 1-66.

Cornago, N. (2010). Perforated sovereignties, agonistic pluralism and the durability of (para)diplomacy. En C. M. Constantinou y J. Der Derian (eds.), Sustainable Diplomacies (pp. 89-108). Basingstoke: Palgrave Macmillan.

Cornago, N. (2013a). Plural Diplomacies Plural diplomacies. Leiden-Boston: Martinus Nijhoff Publishers.

Cornago, N. (2013b, noviembre). (Para)Diplomatic Cultures: Old and New. Documento presentado en el International Workshop Alternative Cultures of Diplomacy, Diplomatic Cultures Research Network, An AHRC-funded Research Network, La Haya, Países Bajos.

Cornago. N. (2016). Diplomacia como heterología: pluralismo social y múltiples mediaciones institucionales en la frontera. En S. González, N. Cornago y C. Ovando (eds.), Relaciones transfronterizas y paradiplomacia en América Latina (pp. 17-45). Santiago: RIL Editores. 
Copeland, D. (2009). Guerrilla Diplomacy: Rethinking International Relations. Boulder: Lynne Rienner.

Dávila, C., Schiavon, J. y Velázquez, R. (2008). La paradiplomacia de las entidades federativas en México. México D.F.: CIDE.

Dean, M. (2010). Governmentality. Power and Rule in Modern Society. Londres: The SAGE Knowledge.

Death, C. (2011). Summit theatre: exemplary governmentality and environmental diplomacy in Johannesburg and Copenhagen. Environmental Politics, 20(1), 119.

Der Derian, J. (1987). On Diplomacy: A Genealogy of Western Estrangement. Oxford: Oxford University Press.

Duchacek, I. D. (1990). Perforated sovereignties: Towards a typology of new actors in international relations. En H. J. Michelmann y P. Soldatos (eds.), Federalism and International Relations: The Role of Subnational Units (pp. 1-33). Oxford: Clarendon Press.

Duran, M. (2016). Paradiplomacy as a diplomatic broker. Diplomacy and Foreign Policy, 1(3), 1-56.

Esposito, R. (28 febrero de 2020). I partiti e il virus: la biopolitica al potere. La Repubblica, s. p. Recuperado de https://bit.ly/3iNBgIg

Foucault, M. (2003). Society Must Be Defended: Lectures at the Collège de France. 1975-76. Nueva York: Picador.

Foucault, M. (2016). Nacimiento de la biopolítica. Madrid: Akal.

Galeotti, M. (2016). Heavy Metal Diplomacy: Russia's Political Use of Its Military In Europe Since 2014. European Council on Foreign Relations, 19 de diciembre. Recuperado de https://ecfr.eu/publication/heavy_metal_diplomacy_russias_ political_use_of_its_military_in_europe_since/

Gely, M. (2016). Hacia una mejor conceptualización teórica de la proyección internacional de los gobiernos locales. En S. González, N. Cornago y C. Ovando (eds.), Relaciones transfronterizas y paradiplomacia en América Latina (pp. 161-175). Santiago: RIL Editores.

Ghilarducci, D. (2020). La diplomacia desde abajo: una herramienta teórica para los estudios globales. Colombia Internacional, (102), 113-138. DOI: 10.7440/colombiaint102.2020.06

Grydehoj, A. (2014). Goals, capabilities, and instruments of paradiplomacy by subnational jurisdictions. En A. Grydehoj (ed.), Local Actions in a Global Context: Paradiplomacy by Subnational Jurisdictions (pp. 10-20). Bruselas: Centre Maurits Coppieters. 
Gobierno Bolivariano de Venezuela. (2020). Plan vuelta a la Patria. [En línea]. Ministerio del Poder Popular para Relaciones Exteriores. Recuperado de http://www.mppre.gob.ve/temas/vuelta-a-la-patria/

Goikoetxea, J. (2017). Demokraziaren pribatizazioa. Donostia: Elkar.

Hardt, M. y Negri, A. (2000). Empire. Cambridge: Harvard University Press.

Heine, J. (2020). ¿Un mundo (y un orden mundial) al revés? En G. L. Gardini (coord.), El mundo antes y después del Covid-19. Reflexiones intelectuales sobre la política, la diplomacia y las relaciones internacionales (pp. 25-29). SalamancaEstocolmo: Instituto Europeo de Estudios Internacionales.

Hocking, B. (1993a). Foreign Relations and Federal States. Londres: Leicester University Press.

Hocking, B. (1993b). Localizing Foreign Policy: Non-Central Governments and Mutilayered Diplomacy. Londres y Nueva York: Macmillan \& St Martin's Press.

Hocking, B. (1996). Between newness and decline: The development of catalytic diplomacy. En J. Melissen (ed.), Innovation in Diplomatic Practice (pp. 21-42). Basingtoke: Palgrave-Macmillan.

Hocking, B., Melissen, J. Riordan, S. y Sharp, P. (2012). Futures for diplomacy, integrative diplomacy in the 21st Century. La Haya: Clingendael, Netherlands Institute of International Relations.

Innerarity, D. (19 de abril de 2020). La complejidad de un virus. La Verdad, sección Opinión, s. p. Recuperado de https://www.laverdad.es/opinion/complejidadvirus-20200419013145-nt.html?ref=https:\%2F\%2Fwww.google.com\%2F

Keating, M. (2013). Regions and international affairs: Motives, opportunities and strategies. En F. Aldecoa y M. Keating (eds.), Paradiplomacy in Action: The Foreign Relations of Subnational Governments (pp. 1-16). Nueva York: Routledge.

Khanna, P. (2011). How to Run the World. Nueva York: Random House.

Kincard, J. (2009). Bibliographic resources for federalism and foreign affairs. En H. J. Michelmann (ed.), Foreign Relations in Federal Countries (pp. 353-383). Montreal: Forum of Federations y McGill-Queen's University Press.

Konrad, M. (2007). International biodiplomacy and global ethical forms: Relations of critique between public anthropology and science in society. Anthropological Quarterly, 80(2), 325-353. DOI: 10.1353/anq.2007.0029

Kuznetsov, A. S. (2015). Theory and Practice of Paradiplomacy: Subnational Governments in International Affairs. Londres: Routledge. 
Lecours, A. (2002). Paradiplomacy: Reflections on the foreign policy and international relations of regions. International Negotiation, 7(1), 91-114. DOI: $10.1163 / 157180602401262456$

Lemke, T. (2017). Introducción a la biopolítica. México: Fondo de Cultura Económica.

Makarychev, A. y Yatsyk, A. (2016). Celebrating Borderlands in a Wider Europe: Nations and Identities in Ukraine, Georgia and Estonia. Baden-Baden: Nomos Verlag.

Malksoo, M. (2012). The challenge of liminality for International Relations theory. Review of International Studies, 38(2), 481-494. DOI: $10.1017 / \mathrm{S} 0260210511000829$

McConnell, F., Moreau, T. y Dittmer, J. (2012). Mimicking state diplomacy: The legitimizing strategies of unofficial diplomacies. Geoforum 43(4), 804-814. DOI: https://doi.org/10.1016/j.geoforum.2012.01.007

Mendiola, I., (2001). Cartografías liminales: el (des)pliegue topológico de la práctica identitaria. Política y Sociedad, 36, 205-221. Recuperado de https://revistas.ucm.es/index.php/POSO/article/view/POSO0101130205A

Metzl, J. F. (2001). Network diplomacy. Georgetown Journal of International Affairs, 2(1), 77-87. Recuperado de https://www.jstor.org/stable/43133985

Paquin, S. (2004). Paradiplomatie et relations internationales: theories des strategies internationals des regions face á la mondialistation. Bruselas: Peter Lang.

Pérez Pirela, M. (7 de agosto de 2020). Entrevista a Dussel: Habla de cómo el COVID19 hackea la modernidad y de su próximo libro sobre estética. Observatorio de Trabajador@s en Lucha. [En línea]. Recuperado de https://observatoriodetrabajadores.wordpress.com/2020/08/10/entrevista-adussel-habla-de-como-el-covid-19-hackea-la-modernidad-y-de-su-proximolibro-sobre-estetica/

REPIT (2020, octubre 29). La heterogeneidad como alternativa al discurso homogeneizador dominante [archivo de video]. Recuperado de https://www.youtube.com/watch?v=QmhvhMW8Ipg

Riordan, S. (2020). El COVID-19 como catalizador. Telesforo Monzón eLab / Euskal Herrigintza Laborategia. [En línea]. Recuperado de https://telesforomonzonlab.eus/el-covid-19-como-catalizador/?lang=es

Rumelili, B. (2012). Liminal identities and processes of domestication and subversion in International Relations. Review of International Studies, 38(2), 495-508. Recuperado de https://www.jstor.org/stable/41485560

Sánchez, V. y Juma, C. (1994). Biodiplomacy: Genetic Resources and International Relations. Nairobi: African Centre for Technology Studies. 
Sánchez, L. (2014). Estudios críticos de fronteras. Aportes de los estudios culturales. $S i$ Somos Americanos. Revista de Estudios Transfronterizos, 14(1), 173-190. DOI: http://dx.doi.org/10.4067/S0719-09482014000100009

Schiavon, J. (2010). Las relaciones exteriores de los gobiernos estatales: el caso de México. En L. Maira (ed.), La política internacional subnacional en América Latina (pp. 135-177). Buenos Aires: Libros del Zorzal.

Selby J. (2007). Engaging Foucault: Discourse, liberal governance and the limits of Foucauldian IR. International Relations, 21(3), 324-345. DOI: 10.1177/ 0047117807080199

Senhoras, E. M. (2009). Geopolítica da paradiplomacia subnacional: Um estudo sobre a extroversão internacional dos municípios da rede de mercocidades. Anales del XII Encuentro de Geógrafos de América Latina (EGAL), del 3 al 7 de abril, Universidad de la República, Montevideo.

Sharp, P. (2009). Diplomatic Theory of International Relations. Cambridge: Cambridge University Press.

Sherr, J. (2013). Hard Diplomacy and Soft Coercion. Londres: Chatham House.

Simón, P. (2001). La Ética de Aristóteles. Albacete: Libros en la Red.

Soldatos, P. (1990). An explanatory framework for the study of Federated States as foreign-policy actors. En H. J. Michelmann y P. Soldatos (eds.), Federalism and International Relations: The Role of Subnational Units (pp. 1-33). Oxford: Clarendon Press.

Sorondo, D. (2019). La representación diplomática y la diplomacia como representación. Puesta en escena del 'orden' y el 'desorden' en la crisis en Ucrania (2013-2015). (Tesis doctoral para obtener el título de doctor en el programa La Globalización a Examen: Retos y Respuestas Interdisciplinares). Universidad del País Vasco/Euskal Herriko Unibertsitatea.

Stengers, I. (2011). Cosmopolitics II. Minneapolis, MN: Minnesota University Press.

Stetter, S. (2016). Middle East diplomacy. En C.M. Constantinou, M. Keer y P. Sharp (eds.), The SAGE Handbook of Diplomacy (pp. 385-387). Londres: SAGE.

Susskind, L., Fuller, B. W. y Fairman, D. (2003). Multistakeholder dialogue at the global scale. International Negotiation, 8(2), 235-266. DOI: $10.1163 / 157180603322576121$

Toro, I. (18 de mayo de 2020), Los secretos del "far west" de la compra de ventiladores de la CPC en China: especuladores corruptos, mercado negro y maletas de billetes. CIPER. [En línea]. Recuperado de https://www.ciperchile.cl/ 2020/05/18/los-secretos-del-far-west-de-la-compra-de-ventiladores-de-la-cpcen-china-especuladores-corruptos-mercado-negro-y-maletas-de-billetes/ 
Tussie, D. (2004). La política comercial en un contexto de federalismo: notas para el estudio del caso argentino. Integración \& Comercio, 21, 69-84.

Vaisse, J. (2007). Transformational diplomacy. Chaillot Paper, 103, junio. París: Insitute for Security Studies European Union.

Villarroel, M. J. (27 de mayo de 2020). Habilitan albergue para venezolanos que permanecían acampando afuera de embajada en Providencia. BiobioChile.cl [En línea]. Recuperado de https://www.biobiochile.cl/noticias/nacional/regionmetropolitana/2020/05/27/habilitan-albergue-venezolanos-permanecianacampando-afuera-embajada-providencia.shtml

Vlavianos-Arvanitis, A. (2005). Bio-diplomacy: An answer to today's global crisis. European Parliament, Simposium en Worldwatch Institute report 'State of the world 2005: Redefining global security', Bruselas, 1 febrero.

Wiseman, G. (2004). 'Polylateralism' and new modes of global dialogue. En C. Jönsson y R. Langhorne, Diplomacy (pp. 36-57). Londres: SAGE.

Zeraoui, Z. (2011). Diplomacia paralela y las relaciones internacionales de las regiones. Desafios, 23(1), 59-96.

Zeraoui. Z. (2016). Para entender la paradiplomacia. Desafíos, 28(1), 15-34. DOI: 10.12804/revistas.urosario.edu.co/desafios/a.4470 